\begin{tabular}{|c|c|}
\hline Title & $\begin{array}{l}\text { Reexamination of } \mathrm{N} \text { composition dependence of coherently grown GaNA s band gap energy with high-resolution x-ray } \\
\text { diffraction mapping measurements }\end{array}$ \\
\hline Author(s) & U esugi, Katsuhiro; Morooka, Nobuki; Suemune, Ikuo \\
\hline Citation & $\begin{array}{l}\text { A pplied Physics Letters, 74(9), 1254-1256 } \\
\text { https://doi.org/10.1063/1.123516 }\end{array}$ \\
\hline Issue Date & 1999-03-01 \\
\hline Doc URL & http:/hdl.handle.net/2115/5575 \\
\hline Rights & $\begin{array}{l}\text { IEEE, A pplied Physics Letters, } 74,9,1999, \mathrm{p} 12541256 \\
\text { @1999 IEEE. Personal use of this material is permitted. However, permission to reprint/republish this material for } \\
\text { advertising or promotional purposes or for creating new collective works for resale or redistribution to servers or lists, } \\
\text { or to reuse any copyrighted component of this work in other works must be obtained from the IEEE." }\end{array}$ \\
\hline Type & article \\
\hline File Information & APL 74-9.pdf \\
\hline
\end{tabular}

Instructions for use 


\title{
Reexamination of $\mathbf{N}$ composition dependence of coherently grown GaNAs band gap energy with high-resolution x-ray diffraction mapping measurements
}

\author{
Katsuhiro Uesugi, ${ }^{\text {a) }}$ Nobuki Morooka, and Ikuo Suemune \\ Research Institute for Electronic Science, Hokkaido University, Sapporo 060-0812, Japan
}

(Received 30 March 1998; accepted for publication 29 December 1998)

\begin{abstract}
GaNAs films grown on $\mathrm{GaAs}(001)$ substrates by metalorganic molecular beam epitaxy were studied by high-resolution $\mathrm{x}$-ray diffraction (XRD) mapping measurements. The lattice constants of epitaxial films are usually estimated from symmetric and asymmetric XRD $2 \theta-\theta$ measurements. In this study, it is pointed out that the consideration of the tilt angle between the GaAs(115) and GaNAs(115) planes caused by elastic deformation of the films is crucial to determine the lattice constants of the GaNAs films coherently grown on GaAs substrates. Mapping measurements of (115) XRD $(2 \theta-\theta)-\Delta \omega$ were performed for this purpose. The band gap energy of the films was determined by Fourier transform absorption spectroscopy measurements. The band gap energy bowing measured up to the $\mathrm{N}$ composition of $4.5 \%$ will be discussed by comparing with other measurements and theoretical calculations. (C) 1999 American Institute of Physics.
\end{abstract}

[S0003-6951(99)00809-8]

GaNAs alloys have been intensively investigated to fabricate new III-V light emitting devices for optical communications. They have peculiar properties compared with conventional III-V alloys, such as large redshift of band gap energy with increasing $\mathrm{N}$ compositions. Weyers, Sato, and Ando ${ }^{1}$ and Kondow et al. $^{2}$ experimentally demonstrated the reduction of band gaps in diluted $\mathrm{GaN}_{x} \mathrm{As}_{1-x}$ alloys ( $x$ $<1.5 \%$ ). GaNAs films were grown on a GaAs substrate by metalorganic chemical vapor deposition (MOCVD) and gassource molecular beam epitaxy (GSMBE), respectively. The measured band gaps were very close to the one calculated by Sakai, Ueta, and Terauchi ${ }^{3}$ based on the dielectric model. ${ }^{4}$ Ougazzaden et al. reported the GaNAs with $3 \% \mathrm{~N}$ composition grown on a GaAs substrate using atmospheric pressure metalorganic vapor phase epitaxy (AP-MOVPE).$^{5}$ They obtained a peak photoluminescence (PL) wavelength of 1.17 $\mu \mathrm{m}$ at room temperature. $\mathrm{Bi}$ and $\mathrm{Tu}$ recently reported the optical behavior of GaNAs films $(x \leqslant 14.8 \%)$ grown on a GaP substrate by GSMBE. ${ }^{6}$ They showed that the band gap bowing was dependent on the $\mathrm{N}$ composition in GaNAs films. We have also reported similar band gap bowing of GaNAs films $(x \leqslant 7.2 \%)$ grown on a GaAs substrate by metalorganic molecular beam epitaxy (MOMBE) ${ }^{7}$

More recently, Bellaiche, Wei, and Zunger demonstrated the dependence of the calculated band gap of GaNAs on the crystalline structures, such as bulk and coherently strained conditions. ${ }^{8}$ They showed the importance of lattice relaxation, chemical effects, and disorder on the band gap of GaNAs. Therefore, it is important to study the detailed strain properties of the GaNAs films. In this study, high-resolution $\mathrm{X}$-ray diffraction (XRD) mapping measurements ${ }^{9,10}$ were carried out to determine the strain properties of the epitaxial GaNAs films and the N composition in GaNAs. Precise lattice structures of the epitaxial films were extracted from

${ }^{a)}$ Electronic mail: uesugi@es.hokudai.ac.jp
(004) $2 \theta-\theta$ scan and two-dimensional $(2 \theta-\theta)-\Delta \omega$ mapping around (115) diffraction peaks as described below. We will discuss the comparison between the measured band gap bowing of the coherently strained GaNAs on (001) GaAs and the previous reports.

GaNAs films were grown on semi-insulating GaAs(001) substrates by MOMBE. The precursors used were triethylgallium, monomethylhydrazine, and trisdimethylaminoarsenic. GaNAs films $0.1-1 \mu \mathrm{m}$ thick were grown at temperatures of $520-570{ }^{\circ} \mathrm{C}$. Detailed growth conditions are reported elsewhere. ${ }^{711}$ The XRD analyses were carried out using a Philips high-resolution XRD system. For the collimator in front of the detector, a $\mathrm{Ge}(220)$ analyzer was used.

In order to determine the lattice parameter of the epitaxial films perpendicular $\left(a_{\perp}\right)$ and parallel $\left(a_{\|}\right)$to the (001) substrate surface, both symmetric and asymmetric reflections are usually measured by the conventional $2 \theta-\theta$ scan method. ${ }^{12-14}$ Figure 1 shows a typical (004) $2 \theta-\theta$ curve of a GaNAs film (optimization of $2 \theta-\theta$ scan is done for the Bragg peak of the GaAs substrate as a reference crystal). A GaNAs peak and some thickness fringe peaks are clearly observed. This suggests that the solid composition is

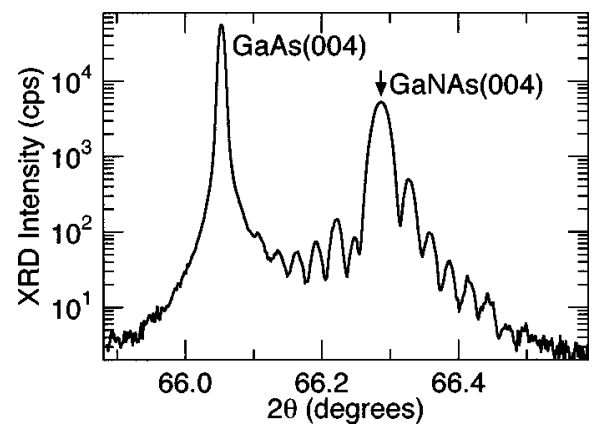

FIG. 1. Typical (004) $2 \theta-\theta$ curve of the GaNAs film grown on the GaAs substrate. The full width at half maximum of the GaAs peak and the GaNAs peak is 17 and 47 arc sec, respectively. 


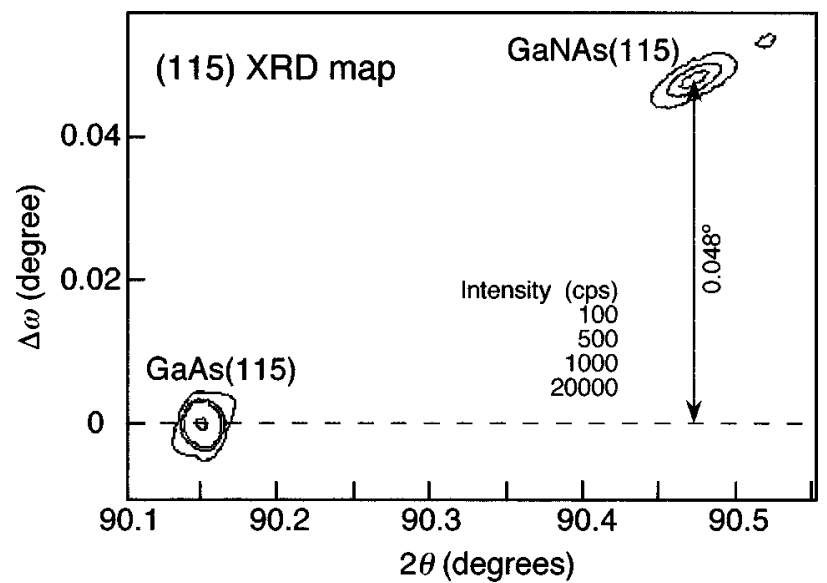

FIG. 2. Typical (115) XRD map of the GaNAs film coherently grown on the $\mathrm{GaAs}$ surface. The tilt angle $\Delta \psi$ between the $\mathrm{GaAs}(115)$ and the GaNAs(115) planes is $0.048^{\circ}$.

homogeneous and the film has a high epitaxial quality. The film tilt between the $\operatorname{GaAs}(001)$ and the GaNAs(001) planes was not observed by the (004) measurement of this sample rotated $180^{\circ}$ around the surface normal. ${ }^{15}$ From the separation between the GaAs and GaNAs peaks, $a_{\perp}$ is estimated to be $5.6356 \AA$. The thickness of the GaNAs film is calculated to be $0.37 \mu \mathrm{m}$ by the usual formula. ${ }^{16}$

It is well known that the inclination between the asymmetric plane of the substrate and that of the epitaxial film is usually observed if the film is subject to a tetragonal distortion. ${ }^{12}$ The angle $\psi$ between the (001) plane and the (115) plane of the strained films is estimated from $\psi$ $=\tan ^{-1}\left(\sqrt{2} a_{\perp} / 5 a_{\|}\right)$. When the lattice structure has the cubic symmetry such as the GaAs substrates or lattice matched or fully relaxed epitaxial films $\left(a_{\perp}=a_{\|}\right), \psi$ is the constant value of $15.793^{\circ}$. However, $\psi$ for the epitaxial films under the elastic distortion is dependent on the lattice parameters $a_{\perp}$ and $a_{\|}$, which suggests that $\psi$ is generally dependent on the strain in the grown epitaxial film. The tilt angle $\Delta \psi$ between the GaAs(115) plane and the GaNAs(115) plane is related through the equation

$$
\Delta \psi=\tan ^{-1}(\sqrt{2} / 5)-\tan ^{-1}\left(\sqrt{2} a_{\perp} / 5 a_{\|}\right) .
$$

Since the conventional $2 \theta-\theta$ scan can measure only in one of the [115] directions of either the substrate or the strained film, a single scan cannot determine the diffraction angle with enough accuracy. $\Delta \psi$ is usually estimated by the two $2 \theta-\theta$ curves with the sample rotated by $180^{\circ}$ around the (115) plane normal. ${ }^{12}$ In this study, high resolution ( $2 \theta$ $-\theta)-\Delta \omega$ mapping methods ${ }^{9,10}$ were used as the characterization technique to determine both the tilt angle $\Delta \psi$ and the actual Bragg angle of the film. The mosaic structure of the films was also observed.

Figure 2 shows a typical $(2 \theta-\theta)-\Delta \omega$ map around the (115) diffraction peaks. This map was constructed by the repeated $2 \theta-\theta$ scans at the different offsets to $\theta(\Delta \omega)$. It can be seen that the diffraction peaks from the GaAs(115) and GaNAs(115) planes are directly observed on the map. The separation of the GaNAs peak in the offset $\Delta \omega$ is estimated to be $0.048^{\circ}$, which corresponds to the tilt angle $\Delta \psi$ between the GaNAs(115) and GaAs(115) planes. From the Bragg

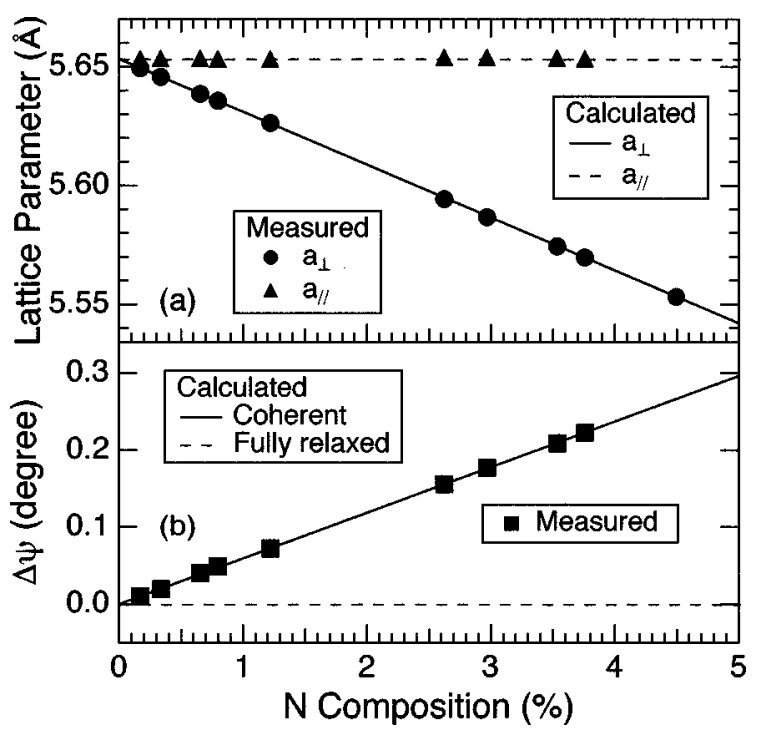

FIG. 3. Dependence of (a) the lattice parameters and (b) the tilt angle $\Delta \psi$ on the $\mathrm{N}$ composition in GaNAs.

angle of the GaNAs(115) plane in Fig. 2, the $d$ spacing of the GaNAs(115) plane $d_{115}$ is estimated to be $1.0848 \AA$. The lattice parameter parallel to the (001) surface $a_{\|}$is related to the above-determined quantities $\left(a_{\perp}, \Delta \psi\right.$ and $\left.d_{115}\right)$ by the equation

$$
1 / d_{115}^{2}=2 / a_{\|}^{2}+25 / a_{\perp}^{2}
$$

and Eq. (1). From these equations, $a_{\|}$is calculated to be $5.653 \AA$, which is in good agreement with the lattice constant of GaAs $\left(a_{\mathrm{GaAs}}\right)$. This shows that the GaNAs film was coherently grown on the GaAs surface. These results show that the asymmetric XRD mapping measurements can precisely determine the three-dimensional crystalline structures of the coherently grown epitaxial films.

The elastic deformation of the epitaxial films is expressed by

$$
\left.a_{\perp}=a_{\|}+\left(C_{11}+2 C_{12}\right) / C_{11}\right)\left(a_{0}-a_{\|}\right),
$$

where $C_{11}$ and $C_{12}$ are the elastic constants for GaNAs and $a_{0}$ is the lattice constant of cubic GaNAs. The N composition $x$ is estimated from $a_{0}$ assuming Vegard's law

$$
a_{0}=x a_{\mathrm{GaN}}+(1-x) a_{\mathrm{GaAs}},
$$

where $a_{\mathrm{GaN}}$ is the lattice constant of cubic GaN (4.50 $\left.\mathrm{A}\right)$. Since the elastic constants for GaNAs are not available, linear interpolation was used for GaAs and cubic phase GaN. ${ }^{17}$ As for the possible bowing of the GaNAs elastic constants, the maximum error for the 5\% $\mathrm{N}$ composition was estimated by assuming the extreme cases of $\mathrm{GaAs}$ and $\mathrm{GaN}$ elastic constants, which were $5.06 \%$ and $4.52 \%$, respectively. The $\mathrm{N}$ composition determined in this way showed good agreements with other data ${ }^{2,5}$ confirmed by the secondary ion mass spectroscopy (SIMS), which will be shown in Fig. 4 later.

Figure 3(a) summarizes the measured lattice parameters of the GaNAs films. The solid and dashed lines are the calculated lattice parameters perpendicular $\left(a_{\perp}\right)$ and parallel $\left(a_{\|}\right)$to the GaAs substrate surface of coherently strained GaNAs. Figure 3(b) shows the corresponding dependence of $\Delta \psi$ on the $\mathrm{N}$ composition measured by (115) XRD maps. Copyright @2001. All Rights Reserved. 


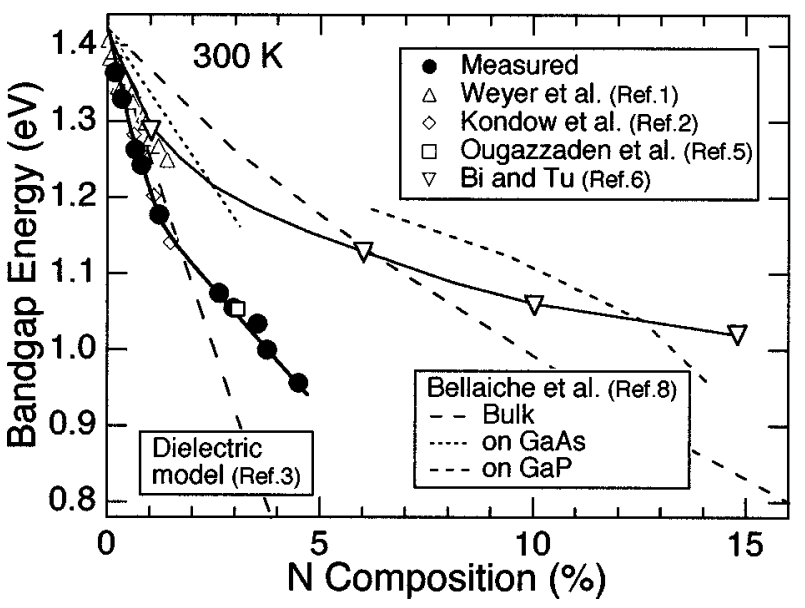

FIG. 4. Relationship between the $\mathrm{N}$ composition in GaNAs and the measured band gap energy. The dashed lines are the calculated band gap energies, based on the dielectric model (Ref. 3) and based on the first-principle supercell models by Bellaiche et al. (Ref. 8).

The solid and dashed lines are calculated from Eq. (1) for coherently strained $\left(a_{\|}=a_{\mathrm{GaAs}}\right)$ and fully relaxed $\left(a_{\perp}=a_{\|}\right)$ GaNAs geometries, respectively. The measured results are in good agreement with the calculations, and this shows that all the samples are coherently grown on the GaAs(001) substrates.

The band gap energy of GaNAs films was estimated by Fourier transform absorption spectroscopy measurements at $300 \mathrm{~K}$. The $\mathrm{N}$ composition dependence of the measured band gap energies is shown in Fig. 4 by the closed circles. All the GaNAs films are coherently grown on (001) GaAs as discussed above. The dashed lines are the theoretical band gap energies, based on the dielectric model ${ }^{3}$ and on the firstprinciple supercell models by Bellaiche, Wei, and Zunger (bulk condition and coherently strained conditions on GaAs and on $\mathrm{GaP}){ }^{8}$ Recent experimental results grown on $\mathrm{GaAs}^{1,2,5}$ and on $\mathrm{GaP}^{6}$ are also shown in this figure. We note that our results agree with the earlier SIMS and PL studies reported by Kondow, Uomi, and Hosomi ${ }^{2}$ and Ougazzaden et al. ${ }^{5}$ The band gap measured on the sample with $4.5 \% \mathrm{~N}$ composition is $0.956 \mathrm{eV}$ at $300 \mathrm{~K}$, which corresponds to the wavelength of $1.3 \mu \mathrm{m}$. This result suggests that coherent GaNAs films with a high quality may be possible to realize long-wavelength laser diodes operating in the $1.3 \mu \mathrm{m}$ region.

The measured band gap energies are very close to the dielectric calculation for the smaller $\mathrm{N}$ compositions $(x$ $\leqslant 1.2 \%$ ). However, they deviate considerably from the dielectric calculation as the $\mathrm{N}$ composition increases. Recently, we reported the MOMBE grown GaNAs properties measured by (004) XRD mapping and atomic force microscopy (AFM) ${ }^{11}$ Although the GaNAs surfaces with small $\mathrm{N}$ compositions were atomically flat across the wide area $(x$ $\leqslant 1.2 \%$ ), those with the higher $\mathrm{N}$ compositions showed the local areas slightly inclined $\left(0.1-0.2^{\circ}\right)$ along the $\langle\overline{1} 10\rangle$ di- rection. The lattice constant of these local tilted GaNAs areas was very close to that of the remaining major GaNAs areas. The deviation of the bowing properties above 2\% in Fig. 4 may be under the influence of this inhomogeneity. Details of this local ordering effect for the higher $\mathrm{N}$ composition are under study. The band gaps of GaNAs films grown on GaP substrates reported by $\mathrm{Bi}$ and $\mathrm{Tu}^{6}$ are closer to the firstprinciple supercell calculations based on the different strain conditions. The deviation of the band gaps of GaNAs grown on GaAs and GaP may be due to the difference of the substrates and the related strain conditions.

In summary, the crystalline structures of GaNAs films grown on $\mathrm{GaAs}(001)$ substrates by MOMBE were studied by high-resolution XRD mapping measurements. To obtain accurate values of the lattice constants of strained epitaxial films, asymmetric XRD mapping measurements should be used. We showed the band gap energy of the coherently strained GaNAs films on the GaAs substrates reaches the wavelength of $1.3 \mu \mathrm{m}$ with the $\mathrm{N}$ composition of $4.5 \%$. Although the measured band gap energy was very close to the dielectric calculation for the smaller $\mathrm{N}$ compositions, it deviated considerably as the $\mathrm{N}$ composition increases. The present measurements as well as others show that there still remain some discrepancies with the first-principle theoretical calculations, and further works will be necessary to have self-consistent understanding.

The authors wish to thank Professor Takayoshi Nakamura for the Fourier transform absorption spectroscopy measurements. They also thank the Trichemical Laboratory Co. for supplying metalorganic precursors. This work was supported in part by a Grant-in-Aid for Encouragement of Young Scientists (No. 09750001) provided by the Ministry of Education, Science and Culture.

\footnotetext{
${ }^{1}$ M. Weyers, M. Sato, and H. Ando, Jpn. J. Appl. Phys., Part 2 31, L853 (1992).

${ }^{2}$ M. Kondow, K. Uomi, K. Hosomi, and T. Mozume, Jpn. J. Appl. Phys., Part 2 33, L1056 (1994).

${ }^{3}$ S. Sakai, Y. Ueta, and Y. Terauchi, Jpn. J. Appl. Phys., Part 1 32, 4413 (1993).

${ }^{4}$ J. A. Van Vechten and T. K. Bergstresser, Phys. Rev. B 8, 3351 (1970).

${ }^{5}$ A. Ougazzaden, Y. Le Bellego, E. V. K. Rao, M. Juhel, L. Leprince, and G. Patriarche, Appl. Phys. Lett. 70, 2861 (1997).

${ }^{6}$ W. G. Bi and C. W. Tu, Appl. Phys. Lett. 70, 1608 (1997).

${ }^{7}$ K. Uesugi and I. Suemune, Jpn. J. Appl. Phys., Part 2 36, L1572 (1997).

${ }^{8}$ L. Bellaiche, S. H. Wei, and A. Zunger, Appl. Phys. Lett. 70, 3558 (1997).

${ }^{9}$ P. F. Fewster, J. Appl. Crystallogr. 22, 64 (1989).

${ }^{10}$ P. F. Fewster, J. Appl. Crystallogr. 25, 714 (1992).

${ }^{11}$ K. Uesugi and I. Suemune, J. Cryst. Growth 189/190, 490 (1998).

${ }^{12}$ A. T. Macrander, G. P. Schwartz, and G. J. Gualtieri, J. Appl. Phys. 64, 6733 (1988)

${ }^{13}$ W. G. Bi, F. Deng, S. S. Lau, and C. W. Tu, J. Vac. Sci. Technol. B 13, 754 (1995).

${ }^{14}$ C. Bocchi, A. Bosacchi, C. Ferrari, S. Franchi, P. Franzosi, R. Magnanini, and L. Nashi, J. Cryst. Growth 165, 8 (1996).

${ }^{15}$ D. K. Bowen and B. K. Tanner, J. Appl. Crystallogr. 28, 753 (1995).

${ }^{16}$ W. T. Stacy and M. M. Janssen, J. Cryst. Growth 27, 282 (1974).

${ }^{17}$ M. E. Sherwin and T. J. Drummond, J. Appl. Phys. 69, 8423 (1991).
} 